AIAA-99-4231

\title{
A COOPERATIVE HUMAN-ADAPTIVE TRAFFIC SIMULATION (CHATS)
}

\author{
Charles T. Phillips* \\ System Resources Corporation \\ Burlington, MA \\ Mark G. Ballin ${ }^{+}$ \\ NASA Langley Research Center \\ Hampton, VA
}

\begin{abstract}
NASA is considering the development of a Cooperative Human-Adaptive Traffic Simulation (CHATS), to examine and evaluate performance of the National Airspace System (NAS) as the aviation community moves toward free flight. CHATS will be specifically oriented toward simulating strategic decision-making by airspace users and by the service provider's traffic management personnel, within the context of different airspace and rules assumptions. It will use human teams to represent these interests and make decisions, and will rely on computer modeling and simulation to calculate the impacts of these decisions. The simulation objectives will be to examine:

- evolution of airspace users' and the service provider's strategies, through adaptation to new operational environments

- air carriers' competitive and cooperative behavior

- expected benefits to airspace users and the service provider as compared to the current NAS

- operational limitations of free flight concepts due to congestion and safety concerns

This paper describes an operational concept for CHATS, and presents a high-level functional design which would utilize a combination of existing and new models and simulation capabilities.
\end{abstract}

\footnotetext{
*Principal Engineer

${ }^{+}$Manager, Aircraft Systems \& Operations, Advanced Air Transportation Technologies project; Senior Member AIAA Copyright (C) 1999 by the American Institute of Aeronautics and Astronautics, Inc. No copyright is asserted in the United States under Title 17, U.S. Code. The U.S. Government has a royalty-free license to exercise all rights under the copyright claimed herein for Governmental purposes. All other rights are reserved by the copyright owner.
}

\section{INTRODUCTION}

\section{Background}

Free flight has been defined by the RTCA Task Force on Free Flight Implementation ${ }^{1}$ as “... a safe and efficient flight operating capability under instrument flight rules in which the operators have the freedom to select their path and speed in real time. Air traffic restrictions are only imposed to ensure separation, to preclude exceeding airport capacity, to prevent unauthorized flight through Special Use Airspace (SUA), and to ensure safety of flight. Restrictions are limited in extent and duration to correct the identified problem."

In the years since the RTCA report, the term "free flight" has been expanded to apply to a variety of proposed air traffic operational concepts. In the context of this report, we will define it as implying future operational concepts with fewer restrictions on airspace user decisions than in today's air traffic control environment. Hereafter, the term "user" or "airspace user" will be used to denote stakeholders who own and operate aircraft, including air carriers, air taxi, general aviation, and military. The "service provider" is the entity which manages air traffic control services, which in the United States is the Federal Aviation Administration (FAA).

The following characteristics are expected under free flight:

- Satellite-based communication, navigation and surveillance (CNS)

- An air traffic management (ATM) system which unifies air traffic control (ATC) and traffic flow management (TFM)

- Cockpit flight information/management systems to support pilots

- Comprehensive decision support systems for controllers

- Collaborative decision-making between airspace 
users and the service provider

- Air traffic control, to the maximum extent feasible, manages by exception rather than by direction

In addition, free flight is expected to produce time savings to passengers and shippers, and to improve service provider efficiency of operation.

\section{Problem Statement}

The aviation community in the United States and increasingly in the world has come to a consensus that free flight is a desirable goal, and policies are being initiated to move from the current air traffic control framework to the new one described above. In particular, the FAA has made free flight the foundation of their air traffic concept of operations to guide future system planning ${ }^{2}$. A large question, however, remains: will free flight work as advertised? In a completely changed air traffic system governed by free flight, with stakeholders having differing objectives which are achieved through interaction (cooperation, competition or a mixture of both), will the promised benefits be realized? In what regions of airspace and under what conditions can free flight be allowed without compromising safety?

To better understand the issues and unforeseen problems that might occur in a free flight environment, NASA has proposed a Cooperative Human-Adaptive Traffic Simulation (CHATS). CHATS will be specifically oriented toward strategic decision-making by users and by the service provider's traffic management personnel, within the context of different airspace and rules assumptions. It will use human teams to represent these interests and make decisions, and will rely on computer modeling and simulation to calculate the impacts of those decisions.

\section{Objectives}

The following objectives were defined for CHATS:

Develop a simulation capability which focuses on user and service provider strategic decision-making in the free flight environment.

The simulation will emphasize strategic as opposed to tactical decisions. Examples of strategic decisions are planning and re-planning flight schedules for a fleet, and type and duration of traffic restrictions. The time horizon for strategic decisions ranges from hours to days to (potentially) years. Examples of tactical situations are collision alerts and avoidance, and changes in arrival sequencing when an aircraft is near the airport.
Assess new roles and strategies for traffic management.

An example of a new strategy for traffic management is to replace the objective of optimal system-wide traffic flow by allowing each air carrier to optimize its own operations, with traffic management assuring system safety.

\section{Test competitive and cooperative strategies.}

The simulation will have differing ground rules. During some runs each user group will execute its own strategy without any communication with others. During other runs, users will share information as they choose. There could be many variations showing different levels of competition and cooperation.

Determine impacts of these strategies upon stakeholders (users and providers).

The simulation will be designed with metrics to measure results such as flight delays, missed connections, airline operating cost impacts, and sector loading.

Find out, during planning and execution of CHATS, the issues important to stakeholders.

Involvement of stakeholders while planning CHATS will allow identification and prioritization of issues and outcomes such as workload, staffing, delays and their associated costs, and fleet utilization. Additional insights will come out of scenario execution.

Permit users and providers to invent and evaluate new strategies.

If full free flight were approved for use today, do the users and service providers have strategies to follow to gain the advantages promised? CHATS will help users and service providers to develop and evaluate such strategies.

Permit providers to study effects of new airspace structures and rules.

From the government perspective, CHATS simulations could test the effect of the abolition of fixed routes as compared with today's web of fixed routes, or other less radical changes in route structure.

\section{CHATS OPERATIONAL CONCEPT}

The operational concept is discussed with respect to potential human actors or players within the simulation, the proposed use of these players, how the simulations will be conducted, and some questions that the evaluations should be designed to answer. 


\section{Players}

The entities involved in operating flights, and supplying players representing their interests, can be divided into air carriers, general aviation, military, and the service provider.

Air Carriers. Air carriers include integrated carriers of passengers and freight, both scheduled and charter, commuter carriers, and air cargo operators including package carriers. Virtually all air carriers have in common the need to plan and schedule fleets of aircraft.

General Aviation. General aviation includes corporate aircraft for executives, and individually owned and piloted aircraft. General aviation does not require the kind of fleet management practiced by air carriers.

Military. Although some military flights are coordinated by an operations center, for example within the Air Mobility Command, most are planned and flown as individual non-scheduled operations similar to those of general aviation.

Service Provider. The service provider is assumed to be a single entity, such as a Civil Aviation Authority or in the United States, the FAA.

\section{Use of Players in the Simulation Environment}

\section{User Teams}

It is proposed that user teams represent the following interests:

Airlines. A small number of airline (including commuter) teams is proposed for CHATS, each of which will include personnel relevant to a given simulation. These normally would include the following:

- Dispatchers (flight re-planning function)

- Scheduling personnel

- Marketing/Fleet Mix decision-makers

who most represent strategic as opposed to tactical decision-makers. The remaining airline personnel including pilots will have their activities represented by a computer simulation.

It will be beneficial to have at least two major competitive airlines represented by teams, to explore their competing and cooperative behavior in future air traffic environments.

Air Cargo Operators. These have distinctive operating procedures from passenger-carrying airlines; for example, night operations and a very focused hub organization. For this reason it would be beneficial to have at least one air cargo operator team involved. The personnel will be similar to those representing airlines.

General Aviation. A general aviation team would represent the interests of individual non-scheduled flights. The team personnel typically would include a corporate owner, a general aviation pilot taking the role of flight planning and re-planning, and perhaps a representative of the Aircraft Owners and Pilots Association (AOPA).

Military. A military team would represent the interests of military flights which use FAA-controlled airspace during peacetime or to support overseas operations. The team personnel typically would include planning specialists from a military operations center, and a pilot performing flight planning and re-planning for individual operations.

\section{Service Provider Teams}

The service provider will be represented by two teams: a traffic management team, and an airspace and rules team. The traffic management team will include traffic managers and ATC operations requirements personnel, as relevant to a given simulation. The airspace and rules team will be represented by FAA specialists in these areas. Other personnel, including air traffic controllers, will have their activities represented by a computer simulation.

\section{Concept Definition and Experiment Design Team}

This team will control the design, conduct, and evaluation of the simulations. It will consist of a simulation policy team, and the airspace and rules team. The policy team will be led by NASA and FAA officials, and will define concepts to be evaluated and establish simulation ground rules.

Figure 1 summarizes the teams. It is anticipated that these teams will be geographically distributed and therefore need to be connected by a wide-area network. A simulation operator will run the simulations and will have a workstation controlling computer simulations, connected by a local-area network.

\section{Simulation Conduct}

The following is a generic description of anticipated simulation conduct. 


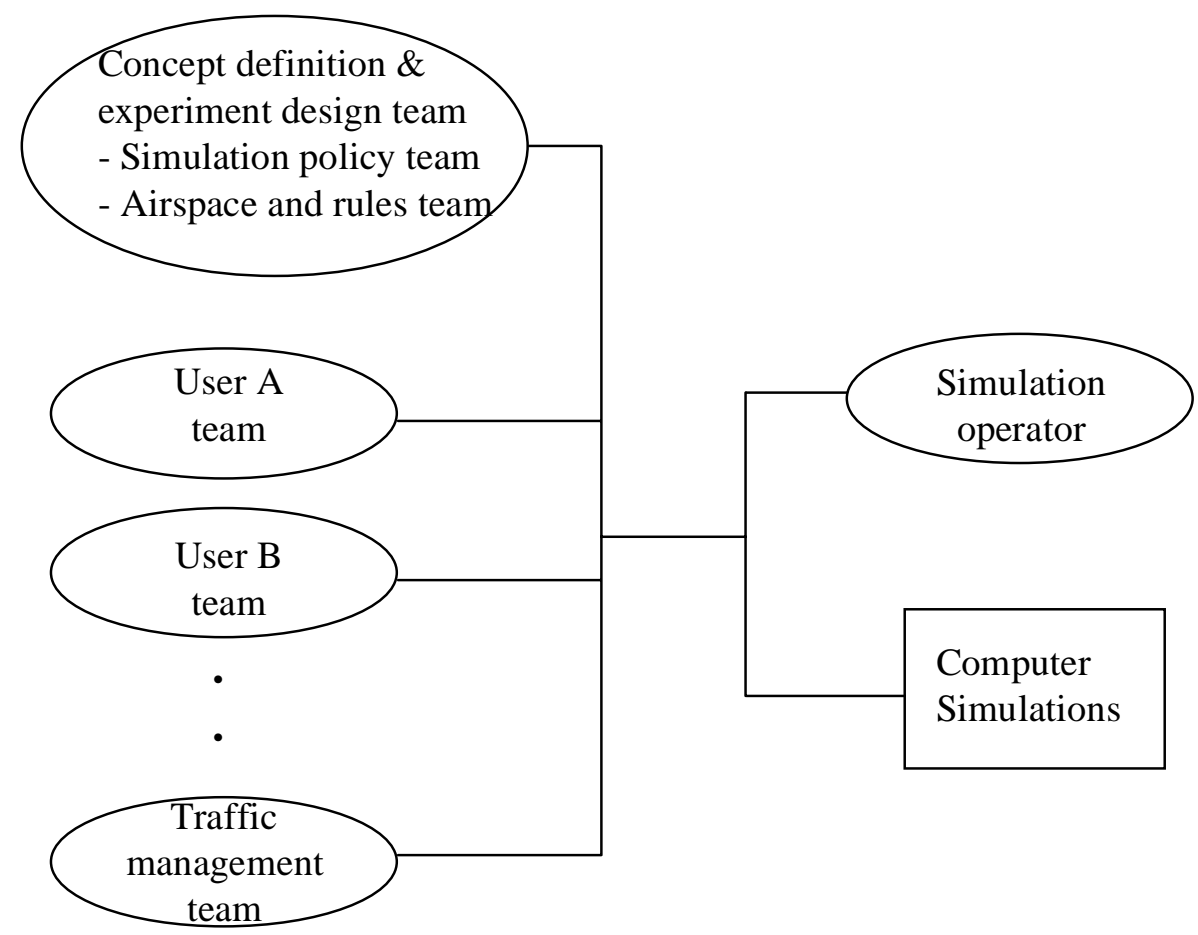

Figure 1. CHATS Teams

The concept definition and experiment design team will coordinate activities of the simulation policy team and the airspace and rules team. The simulation policy team will first define concepts to be evaluated, for example finding the domain of feasibility for a certain free flight concept assuming double today's air traffic volume. It will then establish simulation ground rules, for example whether to allow inter-airline communication and coordination, and defining the speed of onset and extent of a weather front. It will develop a detailed experiment design to carry out a series of simulation runs.

The airspace and rules team will establish a set of airspace and rules assumptions. Route assumptions may allow full freedom of user-preferred routes, all fixed routes, or something in between. The team will define the roles and responsibilities of the traffic management team. It will impose rules such as requiring ground delay programs to take the place of air delays.

User teams will establish their initial strategies. According to the "human-adaptive" principle, these may change as user teams gain experience with the future concepts that are being tested, which may cause interesting and unpredicted effects on the outcomes of the simulation runs.

User teams with scheduled operations will supply initial flight schedules for a day or a week, as the basis of a simulation run, in accordance with the future air traffic environment set by the simulation policy team. For example, if the overall air traffic volume increased by $50 \%$ over the current volume, the team may be directed to submit a schedule with $50 \%$ increased operations over today. The team may also assume service on new flight segments or to new airports as compared with today's schedule. Decision support tools will help in preparing these schedules, and will also be used to support re-planning activities.

Military and general aviation user teams will measure impacts of the simulation on their flights, and summarize these impacts. They will propose to the policy team changes in the simulation ground rules that would benefit their user class.

The traffic management team will set traffic management objectives in coordination with the airspace and rules team. For example, the team may try to maintain system efficiency as in today's operational concept, or they may remove this objective entirely and allow the users to maximize their own individual efficiencies. These objectives may also evolve and change in ways initiated by the learning experience of the team, for the same reason as stated for the user teams. The team will first receive the user-requested 
schedules and combine these with other predetermined schedules and a realistic mix of general aviation and military flights. They will do a "look-ahead" trial simulation run, determine problems and negotiate schedule and flight plan changes with the airlines to avoid sector overloads and to adjust to airport capacities. After this initial adjustment, the traffic simulation will begin.

The simulation will normally run in fast time to conserve time for the players. It can be paused as appropriate to examine problem "events" and decision points for the teams. A classification of such events needs to be determined and would include such items as weather problems and facility saturation. Alternatively, the simulation may be run in real time without pauses. This would force the airline players to react to developing problems in a realistic response manner.

All procedures will be under the control of the simulation operator. The operator will run simulations according to the ground rules, coordinate teams, and collect data in accordance with the experiment design. Adjustments may be made in schedules and flight plans at the pause times to resolve unacceptable situations from the perspective of traffic management or to gain advantage for an airline. Communication systems will allow traffic management - airline coordination and, if allowed under simulation ground rules, inter-airline coordination. Coordination methods from the Collaborative Decision Making (CDM) program, both currently employed and as proposed, will be part of the simulation.

During the simulation run interesting problems may be introduced, following the simulation ground rules and as controlled by the simulation operator. These may be introduced manually or as a result of random processes. These could include current weather (differing from forecast weather) requiring route changes, airport restrictions or closures, establishment of Special Use Airspace (SUA) restrictions, airborne and ATC equipment failures, etc. These will be communicated to the human players and to the computer simulation, leading to the appropriate changes in the simulation run.

\section{Evaluation of Results}

A number of questions will be posed that the simulation will try to answer, for example:

- How will limited airspace and airport resources be allocated in the future, and under increased demand? Are these allocations satisfactory from the point of view of the different stakeholders?

- Is the allocation process stable, including the effects of negotiation, competition, and disturbances, or does it break down?

- How well do future concepts respond to unusual events such as bad weather, a closed runway, or equipment failures?

- Will the airlines get information they need to manage their banks of flights and maintain schedule integrity?

- What kinds of criteria do the teams, representing different stakeholders, use to make decisions?

Metrics will be developed to help compare the results of different simulation runs against each other. The evaluation will be from the perspectives of the service provider (including system effectiveness), and the users.

\section{CHATS FUNCTIONAL DESIGN}

The CHATS functional design is described from several perspectives. First, dataflows are described to show the major modes of communication among the human teams and the models and simulations. Second, communications and operational procedures are discussed. Third, result metrics are defined and discussed.

\section{$\underline{\text { Dataflows }}$}

Six data flow diagrams are shown, organized in a hierarchical manner. In these diagrams the following symbols are used:

- An elliptical box represents a team

- A rectangular box represents a process or model

- A hexagonal box indicates a process external to the principal subject of the diagram

- The circular file storage symbol represents a database or dataset

- A rounded rectangle represents an information display

All major information flows are shown, but in order to avoid clutter not all the external inputs and influences from the player teams are shown.

Figure 2 shows high-level data flow. The major functions shown here, namely Traffic Generation and Tracking Tools, Airport and Terminal Area Queuing Model, Traffic Management Team, and User (Air Carrier) Teams are described in succeeding charts.

The concept definition and experiment design team generates an operations concept and ground rules which are input to traffic management, the user teams, and the 


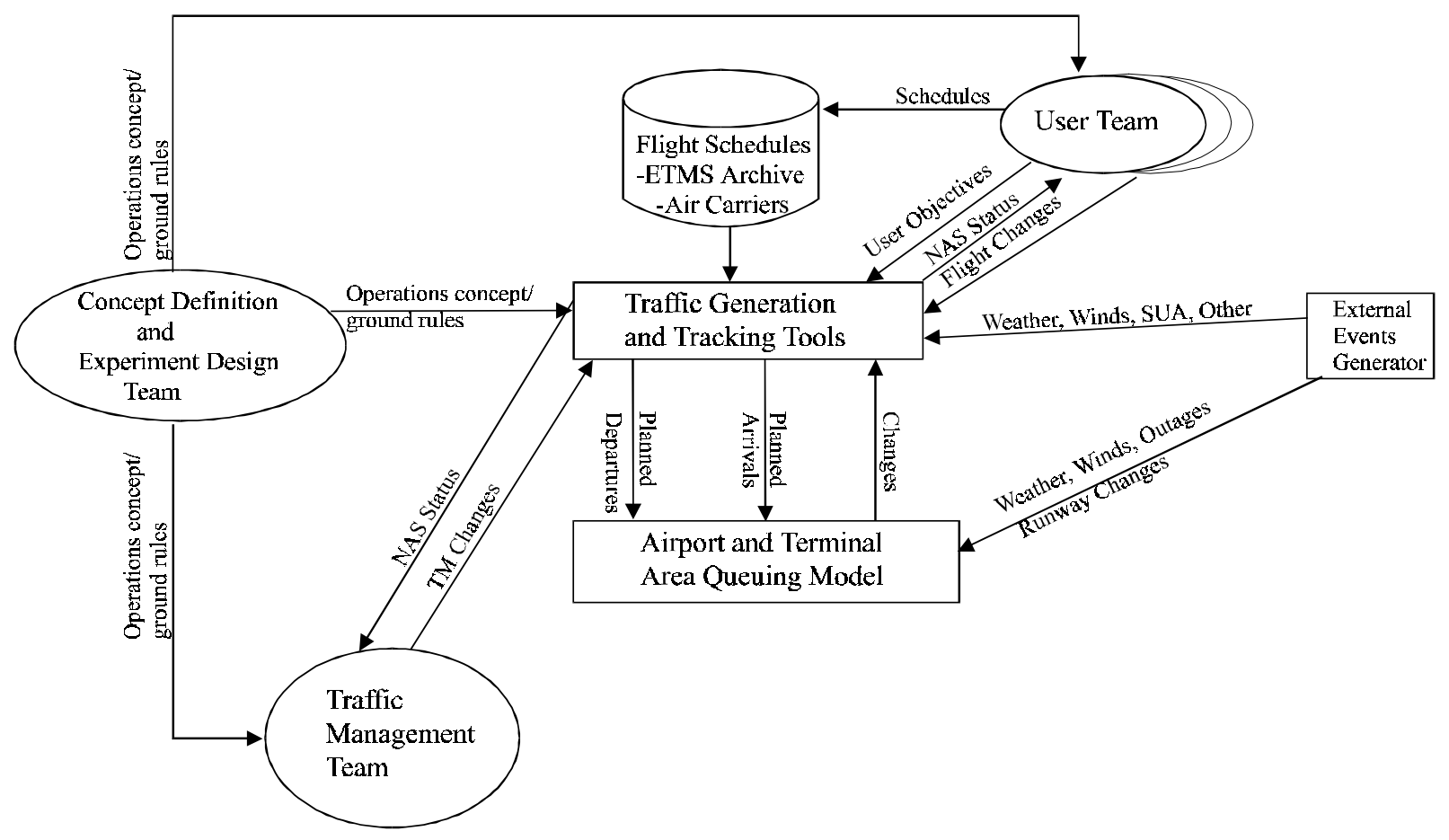

Figure 2. High-Level Data Flow

traffic generation and tracking tools. The simulation operator generates one or more scripts to be followed in the conduct of the simulation which conform to these defined assumptions. Part of the script is the creation of external events such as weather, winds, and SUA use, either by manual parameter setting or by use of a random process. These are also input to the tools.

Flight schedules are provided by air carriers if they are represented in this simulation run as players, otherwise archives from the Enhanced Traffic Management System (ETMS) can be used to fill out a complete schedule. Schedules and user objectives are also input to the tools.

During a simulation run, the tools provide system status both to traffic management and to user teams in a manner similar to what is done in today's CDM environment using the Aircraft Situation Display (ASD) from ETMS and the Flight Schedule Monitor (FSM) display. Based on status, the teams enter changes which will be described in later diagrams.

The Airport and Terminal Area Queuing Model represents the constraints caused by airport demandcapacity imbalances. Weather, winds and airport configuration are the external drivers of airport capacity. The model accepts as inputs planned arrivals and planned departures at capacity-constrained airports, and sends changes back to the tools.

In the following discussion, it should be noted that we are recommending building CHATS using to a great extent existing models and tools as described herein.

Figure 3 shows the Traffic Generation and Tracking Tools. The three principal tools are the Optimized Trajectory Generator (OPGEN), the Total Traffic Tool, and the Find Crossings Tool. These are the three parts of the National Airspace Resource Investment Model (NARIM) ${ }^{3}$. The model was developed for the FAA and has been used by the FAA and the NASA Langley Research Center. Some modifications will have to be made to these tools to adapt them to future air traffic operational concepts.

The OPGEN takes flight schedules and calculates optimized planned trajectories with inputs of SUA, aircraft performance, weather and winds. Operational concept assumptions and user objectives will also influence how the model performs its calculations.

In the real world, the air carrier dispatchers and pilots prepare and file their own flight plans. OPGEN provides realistic trajectories for scheduled flights not represented by air carrier teams. Air carrier teams will 


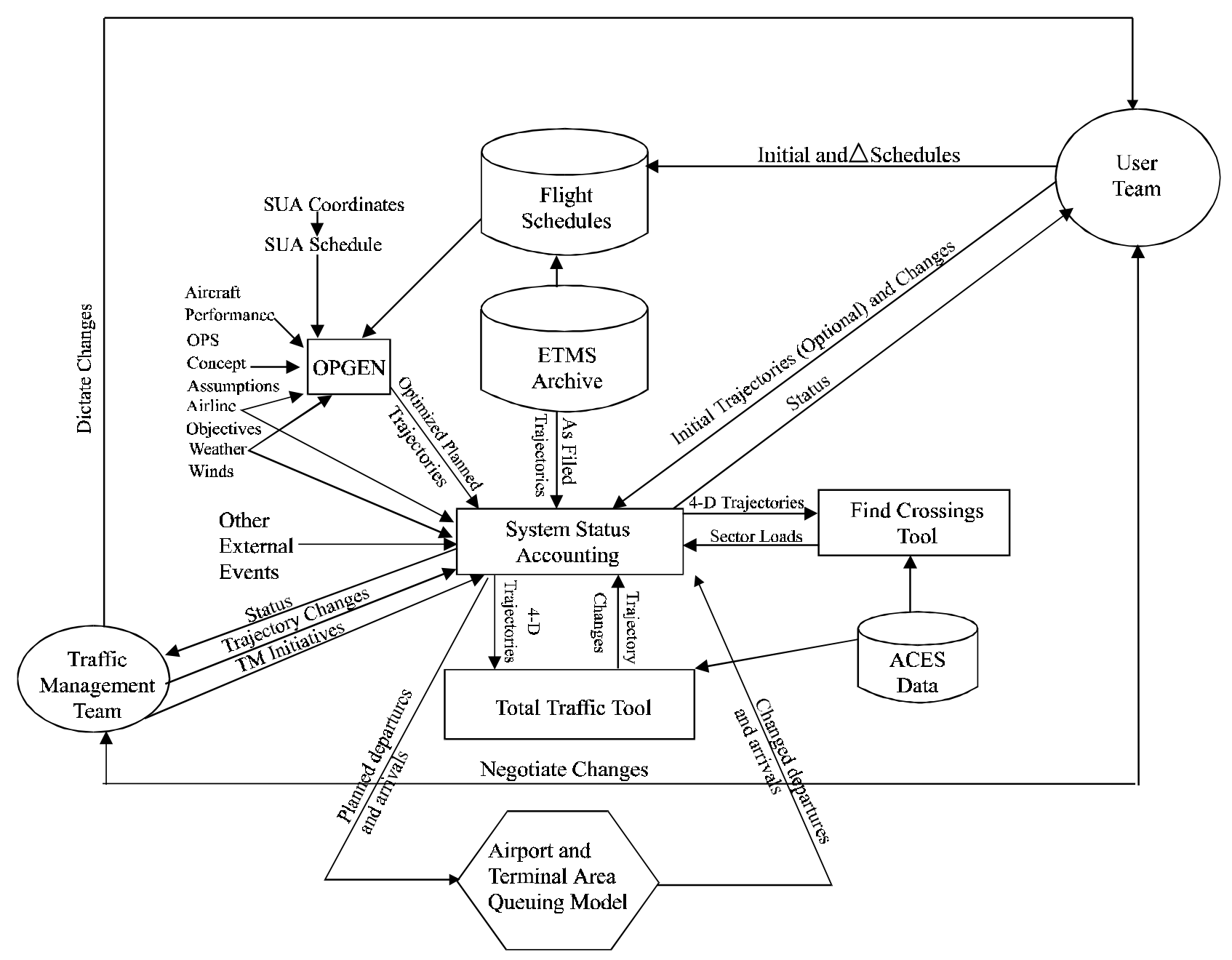

Figure 3. Traffic Generation and Tracking Tools

have the option of directly submitting trajectories based on their own flight plans, or allowing the model to calculate initial trajectories and submit modifications as necessary.

A system status accounting model starts with optimized planned trajectories and adds the trajectories based on filed flight plans for unscheduled flights, including representation of general aviation and military flights, from an ETMS archive. The result is a representation of all flights within the continental U.S. The status model will provide the current state of the National Airspace System to all teams, including flights, weather, airport conditions, etc.

As weather and winds change, OPGEN can be rerun on a regular basis to alter the optimized trajectories from that time forward. Weather has two components in the simulation: forecasts (which can be wrong), and current weather. Other external events such as aircraft problems will change the status. Stochastic disturbances of the trajectories can also be introduced.

The 4-D trajectories are used by the Total Traffic Tool to detect aircraft conflicts. An extension to this tool will estimate delays which result from simulated controller actions to avoid the conflicts. These are fed back to the system status as trajectory changes. The trajectories are also used by the Find Crossings tool to predict sector loads, which become part of the system status. Both of these tools require inputs on the airspace structure from the Adaptation Controlled Environment System (ACES), namely fixes, sectors, and airways. ACES data can be obtained from the ETMS database.

The system status is transmitted to traffic management 
and to the user teams, and these teams will make decisions to cause changes to occur to meet their objectives. The air carrier teams may plan changes to their schedules, generally after negotiation with traffic management. In addition air carriers may input direct trajectory changes based on changed flight plans to meet arrival constraints. If air carriers are not represented as players, traffic management will make changes in flight schedules based on their decision criteria, and they may also input trajectory changes for non-scheduled flights to react to weather and other problems.

Figure 4 further describes the Total Traffic Tool, as modified to account for simulated controller action to avoid aircraft conflicts. The tool finds proximity events, that is aircraft coming to close to each other, from the 4-D trajectories. It then makes an assumption that both aircraft involved in the proximity suffer a standard delay because of the controller's action; it does not model the interaction in detail. These delays are fed back to system status as changed trajectories. The changed trajectories will change future proximity events. Note that a single flight has a good probability of suffering multiple delays over its entire route if that route takes it through multiple areas of heavy traffic.

Figure 5 describes the queuing model. It has an airport configuration and capacity database which has capacity parameters for each capacity-constrained airport. The capacity depends on the runway configuration and meteorological conditions. The runway configuration depends on the winds. The queuing model takes the current capacity and calculates arrival and departure queues based on planned arrival rates and planned departure rates. These queues cause arrival and departure delays which are fed back to the tools.

It is estimated that about 60 airports within the continental U.S. are capacity-constrained and need to be modeled this way. Airport arrival or acceptance rates (AAR) need to be obtained for these airports for each configuration and meteorological condition, and from these a simple capacity model can be developed. Constraints are not anticipated at the other airports because of the expected distribution of traffic.

Two airport queuing models have been created and could be adapted to CHATS: the Approximate Network Delays (AND) model at MIT, and the LMI Capacity Model at the Logistics Management Institute.

Figure 6 shows decision-making within the Traffic Management Team. In CHATS, as fully operational, it is assumed that the traffic management team operates from the System Command Center. A traffic management operations concept is developed and applied in cooperation with the policy team. The system status is input to ETMS/ASD and the Flight Schedule Monitor (FSM) as displays and decision support tools.

Traffic management prepares trial plans, often with the use of the "look-ahead" feature of the tools, involving ground delay programs, changes due to monitor alert, reroutes due to weather, and CDM measures. As previously discussed these changes may be negotiated with the air carriers, schedules may be dictated, or trajectories may be changed.

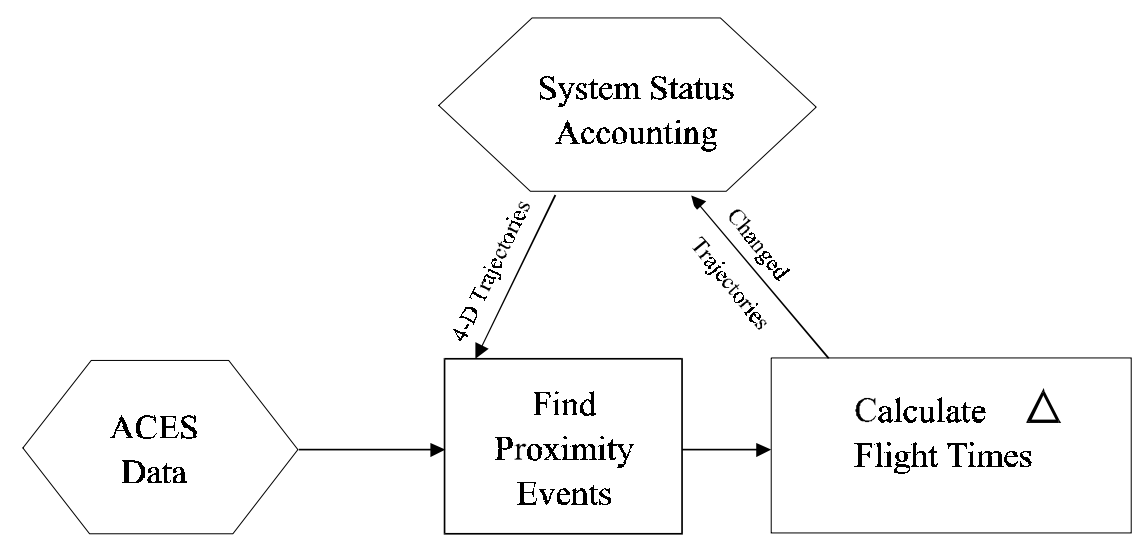

Figure 4. Total Traffic Tool 


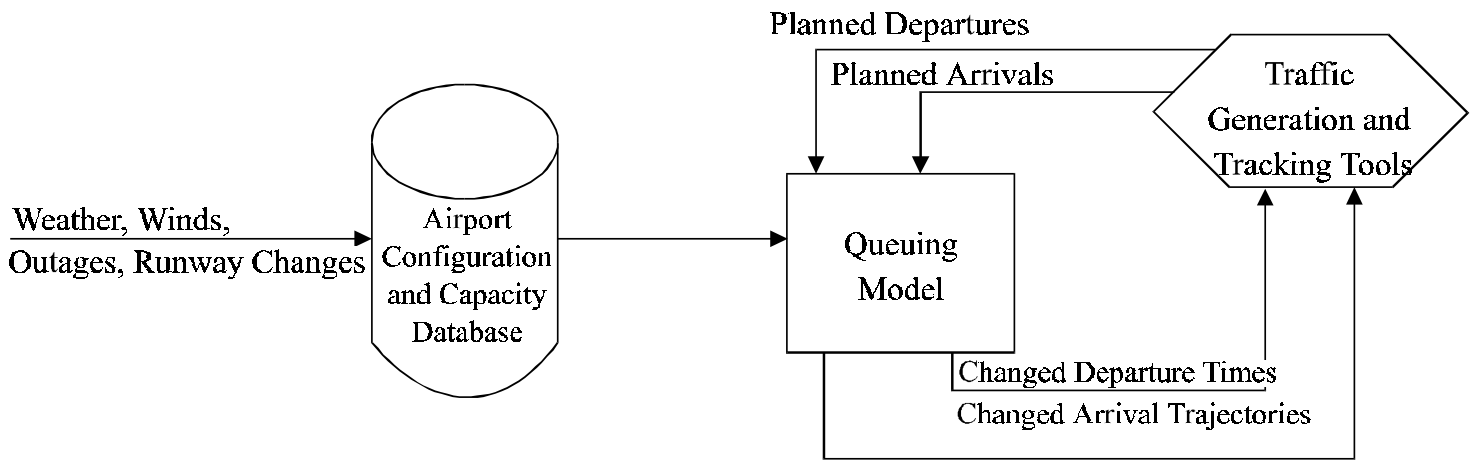

Figure 5. Airport and Terminal Area Queuing Model

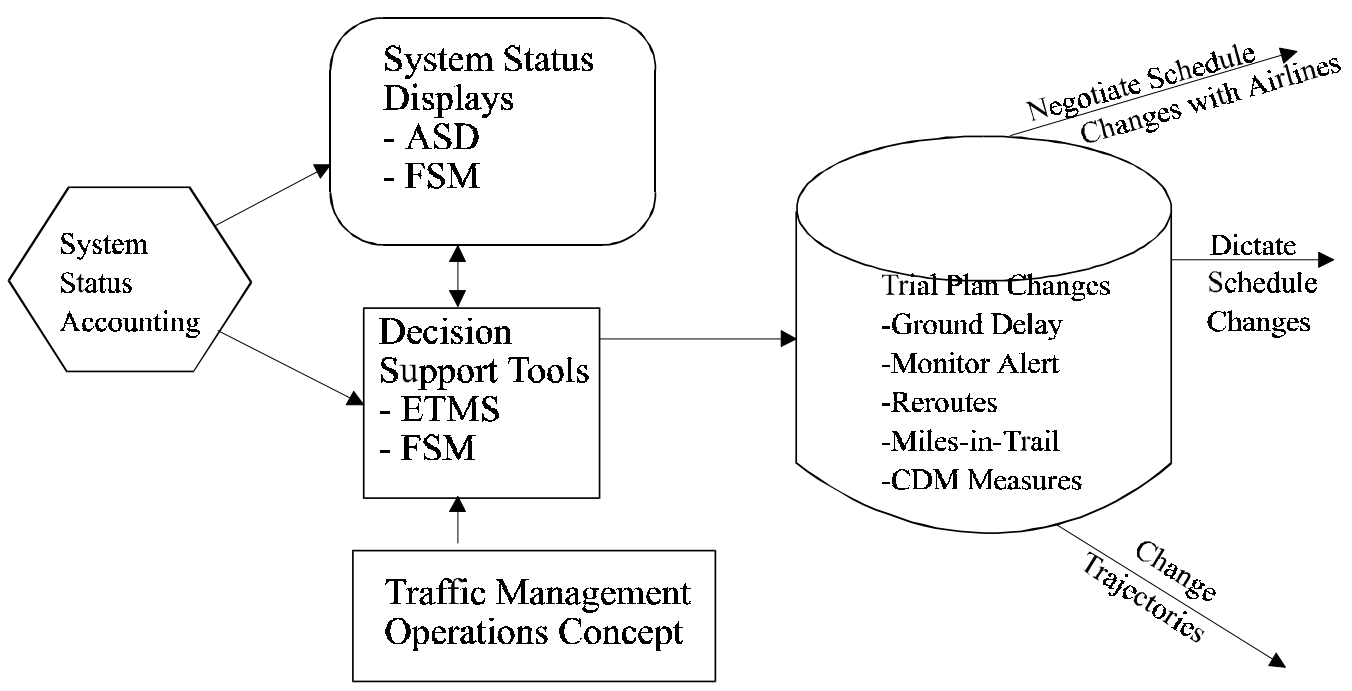

Figure 6. Traffic Management Team

Figure 7 shows decision-making by an air carrier user team. In CHATS, as fully operational, it is assumed that the air carrier teams operate from their AOCs. Team objectives are defined and guide user decisions. The same system status displays as available at traffic management are shown to the air carrier teams, with some information about general aviation, military and competitor air carrier flights removed (this could vary with the ground rules). In addition, an air carrier flight and equipment status database will be created and have an associated data display tool.
Air carrier decisions include a basic weekly schedule, flight/equipment assignments, flight-slot assignments, cancellations, added sections, and earliest departure time estimates. In this simplified simulation environment crew and gate scheduling will not be included. The results of air carrier decisions are negotiated schedule changes with traffic management and, depending on the ground rules, with other user teams; schedule changes input to the flight schedule master database; and trajectory changes from new flight plans. 


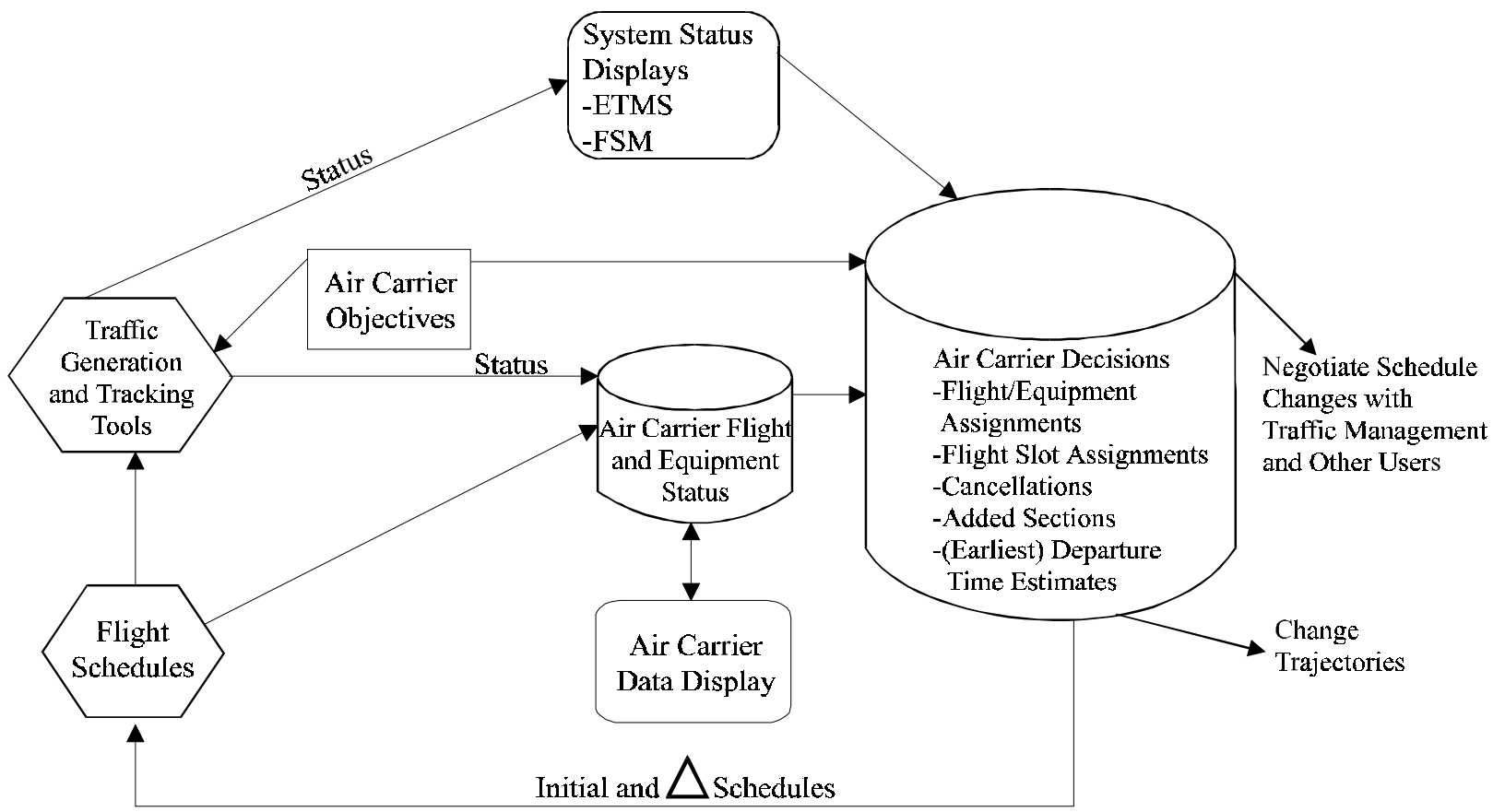

Figure 7. Air Carrier User Team

\section{Communications and Operational Procedures}

Figure 8 illustrates the recommended wide-area communications for the distributed simulation. In this concept, a principal internet service provider (ISP) is employed to provide a secure server for the conduct of the simulation. This server connects the Central Operations Complex's LAN with the Internet, and provides secure delivery services through to all the simulation players. The players can use existing ISPs and connection with adequate bandwidth. The FAA's System Command Center and the AOCs already have or will soon have adequate Internet connections, which are used for example to view independent weather products.

The principal ISP provides three essential services:

- A secure server

- Installation and management

- $24 \times 7$ monitoring

The use of the Internet for the CHATS wide area network will minimize the need for NASA to invest in costly communication equipment and line costs.

An alternative to the model of Figure 8 would be to use the AOCnet, a private internet-like network that connects the AOCs and the FAA facilities for use in
CDM. A connection to this network would have to be made to the Central Operations Complex.

Figure 8 focuses on data communications. Voice and messaging communications also would link the players, either separately or over the Internet.

\section{$\underline{\text { Simulation Result Indicators and Metrics }}$}

Indicators and metrics have been defined for evaluating the results of the simulations. The indicators address outcomes to the maximum extent possible, that is results from the perspective of each stakeholder, and the metrics measure changes in the indicators. If an indicator is too difficult to measure directly, indirect metrics are defined.

The indicators are divided into the following three areas:

- Quantitative indicators related to system operational performance, and of interest to the FAA in NAS oversight;

- Quantitative indicators related to air carrier performance; and

- Qualitative indicators capturing subjective reports by the players concerning strategies used and opinions of different operational concepts. 


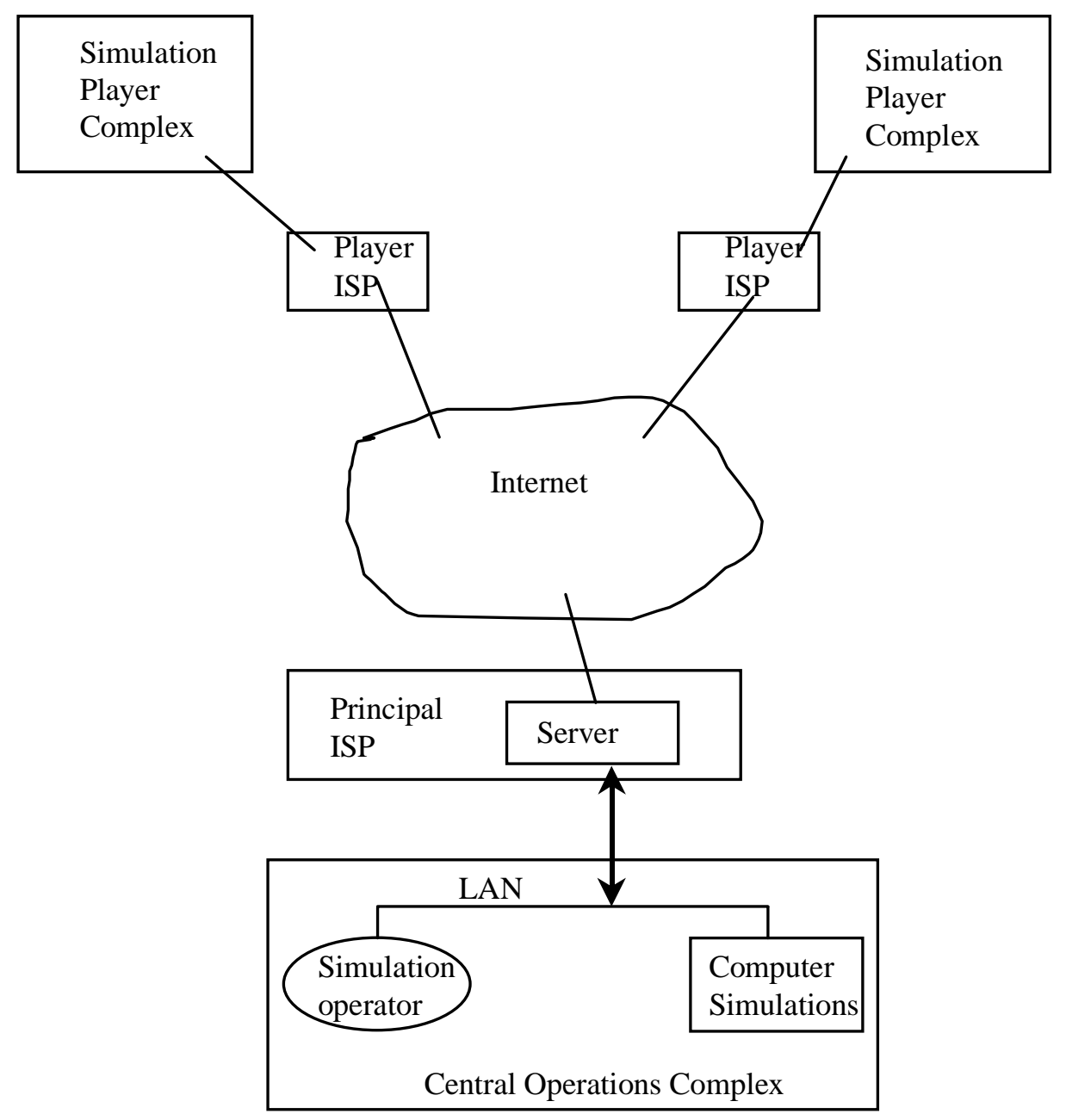

Figure 8. Wide-Area Network Schematic

System Operational Performance Indicators

The FAA's Air Traffic Service ${ }^{4}$ has defined seven indicators of system operational performance. These are Safety, Delays, Flexibility, Predictability, Access, Availability, and Productivity. For all of these except Access, metrics can be defined and evaluated from CHATS.

\section{Air Carrier-Specific Indicators}

Six areas have been proposed for performance from an air carrier perspective ${ }^{5}$. These are Delays, Flexibility, Predictability, Access, Efficiency and Cost. For all except Access, metrics can be defined and evaluated from CHATS.
Qualitative Indicators

A method for eliciting subjective feedback from all the players will be created. The following questions are representative:

- Were there problems in carrying out a scenario? Which of these problems would be likely to occur also in the real world?

- Was the operational concept successful? What problems occurred due to the concept?

- What strategy did your team employ in this scenario?

- What was successful, and what was not, in your carrying out of the strategy?

- Did stakeholder feedback, for example poor achievement of team objective, lead to policy changes for following simulation runs? 


\section{SUMMARY AND CONCLUSIONS}

An operational concept and a functional design have been created for CHATS. The system, and the simulations to be run using it, will focus on aviation user and service provider strategic decision-making in a free flight environment.

CHATS will employ the services of the following decision-makers:

- Air Carriers - to make strategic decisions concerning the management of their fleets, in particular to meet their schedules in the face of obstacles, both prior to and during the conduct of a simulation run.

- Military and general aviation users - to observe impacts on their class of flights and provide feedback to the policy team.

- Traffic management - to make strategic decisions concerning the management of the National Airspace System, in particular resolving bottlenecks and overloads, both prior to and during the conduct of a simulation run.

- Airspace and rules specialists - to create new concepts of organizing the airspace and air traffic rules, consistent with potential future free flight visions, to be utilized in the simulations.

- A simulation policy body - to manage the CHATS resource by creating sets of scenarios which will explore significant future free flight operational concepts.

User and traffic management teams will also serve as human players during the conduct of the simulation runs. The simulation will be under the control of an operator who will create a detailed script for each defined scenario and implement it in a simulation run.

The computer simulation within CHATS will be based on existing air traffic models. NARIM will be used to generate the 4-D aircraft trajectories within the continental United States. ETMS will be used as a data base and presentation tool for part of the system status during the conduct of the simulation. FSM can also be used in the presentation of airport-related information. A queuing model to take account of airport capacity constraints must be developed, or an existing airport queuing model adapted for use. Extensions to NARIM functionality need to be developed along with new system status elements for the players. The new required system and software should be easy to develop given the basic functions which are already in place.

CHATS is designed as a distributed system, with a central operations complex and user teams, traffic management, and potentially other decision-makers using remote workstations at their normal places of business. This will minimize travel time for these personnel. The attention they give to the simulation could be on an as-needed basis. A simulation run would generally be conducted in fast time with pauses to examine the situation and decision points. However, the system will be capable of running a simulation in real time for some defined period, for greater realism.

CHATS will be able to run scenarios which introduce external problems, such as bad weather, airport capacity reductions, SUA activation, and out-of-service aircraft. It will handle initial flight schedules, flight plans, and modifications to flight plans. Air carriers will be able to modify their fleet deployment based on the course of events in the simulation. Negotiation between air carrier and traffic management players, and among air carriers, will be a major activity during a simulation run.

More fundamentally, air carriers will be able to propose wholly revised schedules and show their implications. Also the simulation policy team can introduce new airports or airport expansions.

\section{ACKNOWLEDGMENT}

This work was sponsored by the NASA Langley Research Center under contract NAS2-98005, with support from the NASA Ames Research Center and the Advanced Air Transportation Technologies project.

\section{REFERENCES}

[1] RTCA Inc., Final Report of RTCA Task Force 3, Free Flight Implementation, October 1995.

[2] Federal Aviation Administration, ATS Concept of Operations for the National Airspace System in 2005: Narrative, September 1997.

[3] CSSI Inc., NARIM User Guide, Version 1.1, January 1998.

[4] Federal Aviation Administration, Air Traffic Services Performance Plan for Fiscal Years 1997-1999, 1997.

[5] CNS/ATM Focused Team, Report of the Air Traffic Services Performance Focus Group, October 1998. 